
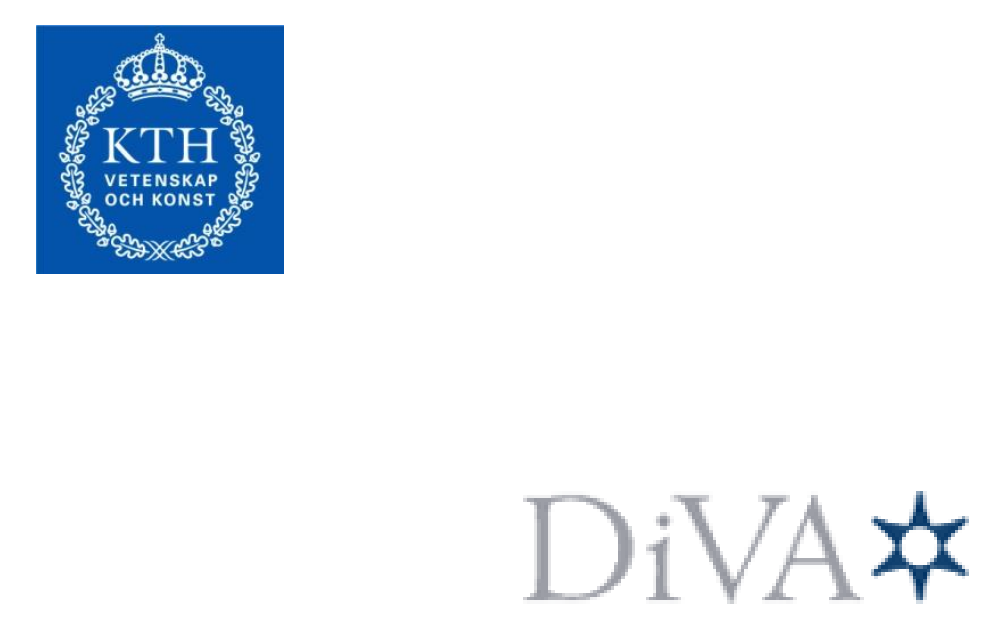

http://kth.diva-portal.org

This is an author produced version of a paper published in 19th IEEE International Conference on Electronics, Circuits and Systems (ICECS 2012).

This paper has been peer-reviewed but does not include the final publisher proofcorrections or proceedings pagination.

(C) 2012 IEEE. Personal use of this material is permitted. Permission from IEEE must be obtained for all other uses, in any current or future media, including reprinting/republishing this material for advertising or promotional purposes, creating new collective works, for resale or redistribution to servers or lists, or reuse of any copyrighted component of this work in other works.

Citation for the published paper:

Sha Tao, Julian Garcia, Saul Rodriguez and Ana Rusu.

Analysis of Exponentially Decaying Pulse Shape DACs in Continuous-Time SigmaDelta Modulators.

19th IEEE International Conference on Electronics, Circuits and Systems (ICECS 2012). Access to the published version may require subscription.

Published with permission from: IEEE 


\title{
Analysis of Exponentially Decaying Pulse Shape DACs in Continuous-Time Sigma-Delta Modulators
}

\author{
Sha Tao, Julian Garcia, Saul Rodriguez and Ana Rusu \\ School of Information and Communication Technology (ICT) \\ KTH Royal Institute of Technology \\ SE-164 40 Kista, Sweden \\ Email:\{stao,julianmg,saul,arusu\}@kth.se
}

\begin{abstract}
The performance of continuous-time (CT) sigmadelta $(\Sigma \Delta)$ modulators is severely degraded by the clock jitter induced timing variation in their feedback digital-to-analog converters (DACs). To mitigate this non-ideality, jitter sensitivity reduction techniques that employ exponentially decaying pulse shape DACs have been recently reported. In this paper, exponentially decaying DACs are investigated and generalized expressions are derived. In addition, another exponentially decaying DAC is proposed, which can potentially achieve both good jitter immunity and amplitude efficiency. To validate the theoretical results, the proposed DAC, together with other exponentially decaying DACs, are employed in a $3^{\text {rd }}$ order 1-bit CT $\Sigma \Delta$ modulator test case and evaluated through behavioral simulations.
\end{abstract}

\section{INTRODUCTION}

During the past decade, CT $\Sigma \Delta$ ADCs have received a growing interest due to their superior speed/power trade-off over their discrete-time (DT) counterparts [1]. However, one critical non-ideality limiting their performance is the high sensitivity to the clock jitter in the feedback DACs. Clock jitter introduces timing variation to the feedback DAC and consequently induces a statistical integration error. This error, in return, increases the noise floor and, hence, degrades the modulator's signal-to-noise-ratio (SNDR). One way to reduce this error is through minimizing the amplitude of the tail feedback pulse, as the amount of integration error depends on the pulse shape of the DAC's feedback signal. Following this strategy, feedback DACs that employ exponentially decaying pulse shape have been proposed in literature. Instead of using a traditional rectangular pulse shape, e.g., the nonreturn-to-zero (NRZ) feedback (Fig. 1(a)), [2] proposes an exponentially decreasing feedback pulse (Fig. 1(b)) implemented by the switched-capacitor-resistor (SCR) architecture. However, the DAC feedback peak current is largely increased so as to transfer the same amount of charge in one clock period. Consequently, the SCR feedback imposes stringent speed requirements on the $1^{\text {st }}$ integrator. Based on the SCR DAC architecture, several follow-up works have been reported lately: the switched-capacitor switched-resistor (SCSR) [3] (Fig. 1(c)), the full clock period SCR (FSCR) [4] (Fig. 1(d)), and the dual SCR (DSCR) [5] (Fig. 1(e)) architectures. Among them, [3] and [5] focus on relaxing the slew-rate requirement of the amplifier in the $1^{\text {st }}$ integrator; while [4] mainly aims to further reduce the jitter induced noise. In this paper, a full clock period SCSR (FSCSR) DAC feedback waveform is
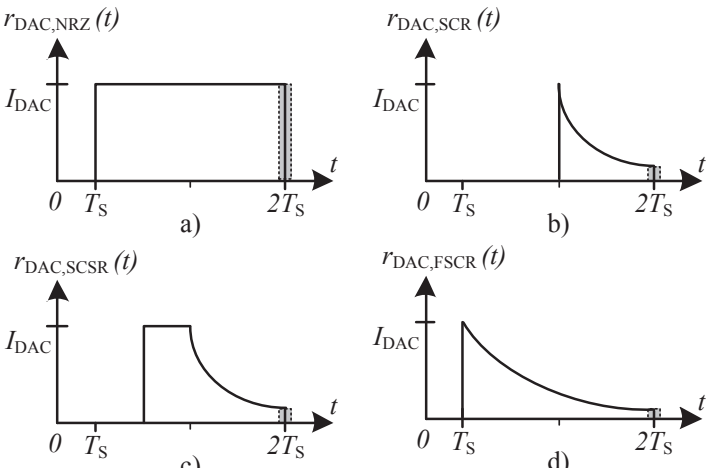

c)
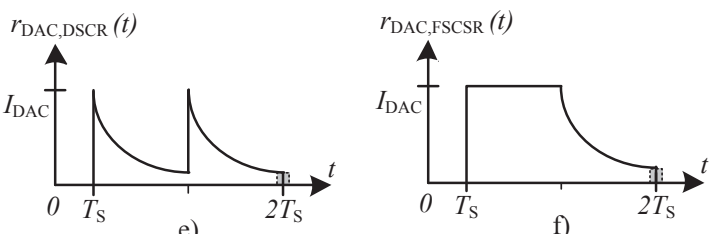

Fig. 1. Normalized impulse response of feedback DACs with single edge jitter. a) non-return-to-zero (NRZ), b) switched-capacitor-resistor (SCR), c) switched-capacitor switched-resistor (SCSR), d) full clock period SCR (FSCR), e) dual SCR (DSCR), f) full clock period SCSR (FSCSR).

proposed, as illustrated in Fig. 1(f). The proposed waveform is shaped in a way that can transfer feedback charge during the whole clock period $T_{S}$ while starts decaying after the half clock period. By doing so, this FSCSR DAC can potentially achieve similar jitter immunity performance as the traditional SCR without significantly increasing the DAC output peak current amplitude. It is worth to mention that even though only the single-bit case is treated here, the proposed waveform can be easily applied to multi-bit DACs.

\section{FSCSR DAC OPERATION}

Fig. 2 depicts one possible implementation of the proposed FSCSR feedback DAC, assuming a $G_{m}-\mathrm{C}$ based integrator. As it can be appreciated from the figure, the DAC has two replicated circuit cells, which are time-interleaved in order to achieve full clock period charge transferring. The operation of this DAC can be divided into three phases controlled by $\Phi_{1}$, $\Phi_{2}, \Phi_{3}$ and $\Phi_{4}$. For simplicity, the operation of the two cells will be explained together in the fashion of "Cell I (Cell II)". In the precharge phase, which occurs during $\Phi_{1}\left(\Phi_{2}\right)$, the bottom 

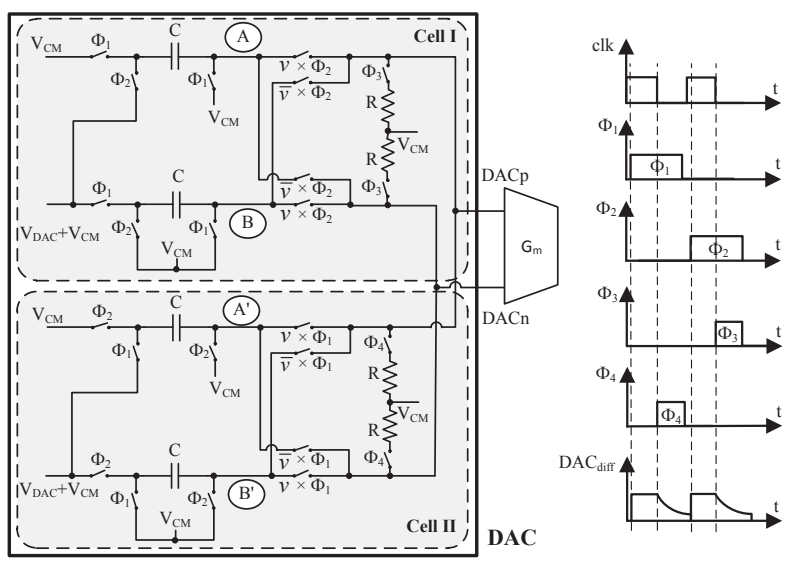

Fig. 2. Schematic and timing diagram of the FSCSR DAC.

capacitor samples the voltage $V_{D A C}$, while the top capacitor is discharged by connecting its plates to $V_{C M}$. In the constant charge phase, which occurs during $\Phi_{2} \cdot \bar{\Phi}_{3}\left(\Phi_{1} \cdot \bar{\Phi}_{4}\right)$, the voltage of nodes $\mathrm{A}\left(\mathrm{A}^{\prime}\right)$ and $\mathrm{B}\left(\mathrm{B}^{\prime}\right)$ are raised to $V_{D A C}+V_{C M}$ and $V_{D A C}-V_{C M}$, respectively. These nodes are connected to the DAC output $D A C_{p}$ and $D A C_{n}$ via the switches controlled by the quantizers output, $v$, which determines the polarity of the differential output, $D A C_{d i f f}=D A C_{p}-D A C_{n}$. The charge on $D A C_{p}$ and $D A C_{n}$ are then kept constant until next phase. In the exponential discharge phase, which occurs during $\Phi_{2} \cdot \Phi_{3}$ $\left(\Phi_{1} \cdot \Phi_{4}\right)$, both top and bottom capacitors are discharged to $V_{C M}$ via resistors through the switches controlled by $\Phi_{3}\left(\Phi_{4}\right)$. Since the FSCSR architecture has two replicated cells, the silicon area is potentially doubled in the DAC. However, there is no power penalty introduced as the two circuit cells operate alternatively. It is worth noticing that matching between the two time-interleaved cells needs to be carefully treated, so as to avoid introducing much noise.

\section{TheORETICAL AnAlysis}

As introduced in section I, the most critical impact of clock jitter on CT $\Sigma \Delta$ modulators is the timing variation in the DAC feedback pulse. The outermost branch DAC will have the most detrimental effect on the modulator's performance, as no noise shaping will take place. The impact of jitter can be further divided into two categories: 1) a random change in pulse position, and 2) a random change in pulse width. It has been shown that the latter one is more critical than the former one [7], thus, this work will focus on analyzing only the pulse width (PW) jitter. The PW jitter induced sampling uncertainty $t_{j}$ can be assumed as zero mean Gaussian distributed with variance $\sigma_{j}^{2}$. The PW jitter induced noise $N_{\sigma_{j}}$ that enters in the outermost branch DAC (before its scaling coefficient) can be expressed as [6]:

$$
N_{\sigma_{j}} \approx \frac{\sigma_{e, \mathrm{SE}}^{2} \cdot A}{T_{S}^{2}},
$$

where $T_{S}$ is the clock period, $\sigma_{e, \mathrm{SE}}^{2}$ is the variance of singleended jitter induced charge error $\epsilon_{j, \mathrm{SE}}$, and $A$ is the activity factor that refers to the number of jittered edges in one $T_{S}$.
The single-ended jitter induced charge error $\epsilon_{j, \mathrm{SE}}$, which applies to all the DACs in Fig. 1, can be expressed by:

$$
\begin{aligned}
\epsilon_{j, \mathrm{SE}}= & \int_{\alpha T_{S}}^{\gamma T_{S}} r_{D A C}(t) d t+\int_{\gamma T_{S}}^{\beta T_{S}+t_{j}} r_{D A C}(t) d t \\
& -\int_{\alpha T_{S}}^{\beta T_{S}} r_{D A C}(t) d t-\int_{\gamma T_{S}}^{\beta T_{S}} r_{D A C}(t) d t,
\end{aligned}
$$

where $r_{D A C}(t)$ is the DAC feedback impulse response, $\alpha$ and $\beta$ are the normalized starting and ending time instants of the corresponding DAC pulse, and $\gamma$ is the normalized time instant when the exponential decaying begins. In this calculation, $\gamma$ is assumed as jitter free. This assumption will be further discussed in section III. By applying a $2^{\text {nd }}$ order Taylor expansion, (2) can be solved as:

$$
\begin{aligned}
\epsilon_{j, \mathrm{SE}} & =-P \cdot I_{D A C} \cdot \tau\left(\mathrm{e}^{-\frac{(\beta-\gamma) T_{S}+t_{j}}{\tau}}-\mathrm{e}^{-\frac{(\beta-\gamma) T_{S}}{\tau}}\right) \\
& =P \cdot I_{D A C} \cdot \mathrm{e}^{-\frac{(\beta-\gamma) T_{S}}{\tau}} t_{j},
\end{aligned}
$$

where $I_{D A C}$ is the normalized peak amplitude of each DAC pulse, while $P$ denotes the pulse transition amplitude factor which equals 2 for NRZ and 1 for all the others. Also for NRZ, $\gamma=\beta$ and $\tau \rightarrow \infty$. Then, $\sigma_{e, \mathrm{SE}}^{2}$ can be derived as:

$$
\begin{aligned}
\sigma_{e, \mathrm{SE}}^{2} & =\int_{t=-\infty}^{+\infty}\left[\left(\epsilon_{j, S E}-E\left[\epsilon_{j, S E}\right]\right)^{2} f(t)\right] d t_{j} \\
& =\left(P \cdot I_{D A C}\right)^{2}\left(\mathrm{e}^{-\frac{(\beta-\gamma) T_{S}}{\tau}}\right)^{2}{\sigma_{j}}^{2},
\end{aligned}
$$

where $f(t)$ is the probabilty density function (PDF) of $t_{j}$, and $E\left[\epsilon_{j, \mathrm{SE}}\right]$ is the expected value of $\epsilon_{j, \mathrm{SE}}$. It can be appreciated from (4) that the single-ended jitter induced error of SCR, DSCR, SCSR and FSCSR DACs are essentially equal as long as the decaying starts at the same time instant $\gamma$ with the same time constant $\tau$.

Recalling from (1), $\sigma_{e, S E}^{2}$ can be used to calculate $N_{\sigma_{j}}$ by relating it to $T_{S}$ and the corresponding activity factor $A$. Moreover, to calculate the jitter induced in-band-noise, $\mathrm{IBN}_{\sigma_{j}}$, the noise entering in the outermost branch DAC, $N_{\sigma_{j}}$, needs to be further referred to the modulator input.

$$
\mathrm{IBN}_{\sigma_{j}} \approx \frac{a_{1}^{2} N_{\sigma_{j}}}{b_{1}^{2} \mathrm{OSR}}=\left(\frac{a_{1}}{b_{1}}\right)^{2} \frac{\sigma_{e, \mathrm{SE}}^{2}}{T_{S}{ }^{2}} \frac{A}{\mathrm{OSR}},
$$

where the oversampling ratio (OSR) and $T_{S}$ are equal for all the DACs. $A \approx 0.7$ for NRZ and $A=2$ for all the other DACs. $a_{1}$ is the feedback coefficient of the outermost DAC and $b_{1}$ is the feedforward coefficient from the modulator input. Here, all DAC pulses are assumed to transfer the same amount of feedback charge per clock period. We can appreciate from (5) that the jitter induced in-band-noise (IBN) of each DAC feedback is not only dependent on its charge error variance but also on its feedback coefficient. For better comparison, two performance metrics are applied in this work, namely amplitude efficiency [3], $\eta_{a}$, and jitter immunity, $\eta_{j}$. 
The amplitude efficiency, $\eta_{a}$, measures the ratio of the charge transfered over one clock period between a NRZ pulse and an exponentially decaying pulse:

$$
\eta_{a}=\frac{H_{\mathrm{NRZ}}(z)}{H_{\exp }(z)}=\frac{a_{1, \exp }}{a_{1, \mathrm{NRZ}}}
$$

where $a_{1 \text { exp }}$ denotes the feedback coefficient of each exponentially decaying DAC, and $a_{1, \mathrm{NRZ}}$ denotes the feedback coefficient of the NRZ DAC. $H_{\exp }(z)$ and $H_{\mathrm{NRZ}}(z)$ are their DT equivalent transfer functions which are derived using the impulse-invariant transformation [1]. The DT equivalent transfer functions of all the DACs can be generalized by a single expression as:

$$
H(z)=-\frac{\left(\alpha+\tau \mathrm{e}^{-\frac{(\beta-\gamma)}{\tau}}-\gamma-\tau\right) R}{z-1},
$$

where $R$ is the repeating factor which denotes the number of times the DAC pulse waveform is repeated over one period. $R=2$ for the DSCR pulse and $R=1$ for all the others. Given $H(z)$, the amplitude efficiency of each DAC can be obtained by using (6). Naturally, $\eta_{a, \mathrm{NRZ}}=1$. The resultant $\eta_{a}$ for all the other DACs can be generalized as:

$$
\eta_{a, \exp }=-\frac{1}{\left(\alpha+\tau \mathrm{e}^{-\frac{(\beta-\gamma)}{\tau}}-\gamma-\tau\right) R} .
$$

The jitter immunity, $\eta_{j}$, measures how much the jitter sensitivity is reduced in a exponentially decaying pulse as compared to a NRZ pulse:

$$
\eta_{j}=10 \log _{10}\left(\frac{\mathrm{IBN}_{\sigma_{j}, \exp }}{\mathrm{IBN}_{\sigma_{j}, \mathrm{NRZ}}}\right),
$$

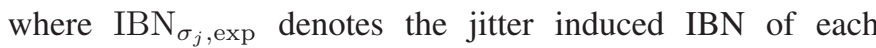
exponentially decaying DAC, while $\mathrm{IBN}_{\sigma_{j}, \mathrm{NRZ}}$ denotes the jitter induced IBN of the NRZ DAC. It can be seen from (6) that $\eta_{a}$ is linearly related to $a_{1, \exp }$. By combining (4), (5) and (6), an alternative way to express $\mathrm{IBN}_{\sigma_{j}}$ can be found:

$$
\mathrm{IBN}_{\sigma_{j}}=\eta_{a}^{2} \frac{A}{\mathrm{OSR}} \frac{I_{D A C}^{2}\left(\mathrm{e}^{-\frac{(\beta-\gamma) T_{S}}{\tau}}\right)^{2} \sigma_{j}^{2}}{T_{S}{ }^{2}}
$$

Given $\mathrm{IBN}_{\sigma_{j}}$, the jitter immunity of each DAC can be obtained from (9). Naturally, $\eta_{j, \mathrm{NRZ}}=0 \mathrm{~dB}$. The resultant $\eta_{j}$ for all the other DACs can hence be generalized as:

$$
\eta_{j, \exp }=-\frac{5}{7} \eta_{a, \exp }^{2}\left(\mathrm{e}^{-\frac{(\beta-\gamma) T_{S}}{\tau}}\right)^{2} .
$$

In Fig. 3 and Fig. 4, the amplitude efficiency $\eta_{a}$ given by (8) and the jitter immunity $\eta_{j}$ given by (11) are plotted as functions of normalized $\tau$. For a fair comparison, $\gamma$ is fixed as $0.5 T_{S}$ for SCR, SCSR and FSCSR. Also noted that here $\gamma=0$ for FSCR and DSCR, $\alpha=0.25 T_{S}$ for SCSR, $\beta=0.5 T_{S}$ for DSCR. As it can be appreciated from Fig. 3, the proposed FSCSR pulse shape has better amplitude efficiency than any other exponentially decaying pulse shapes, especially the SCR and FSCR. On the other hand, Fig. 4 suggests that the FSCSR DAC also has better jitter immunity compared to the other exponentially decaying DACs, except the FSCR.

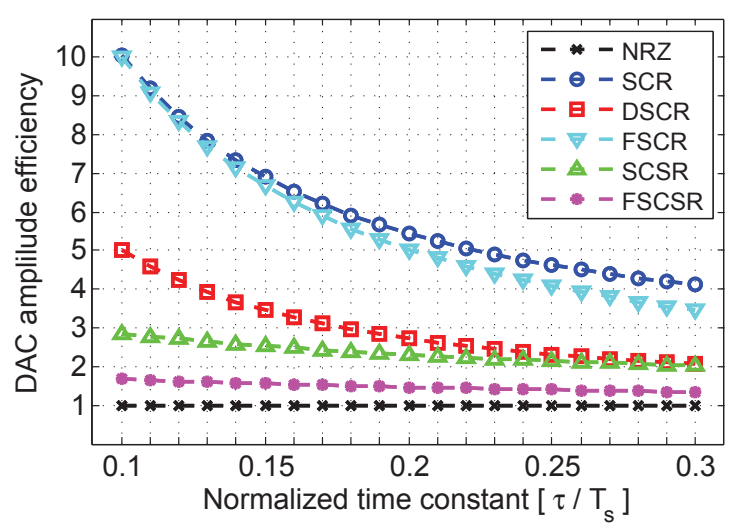

Fig. 3. The amplitude efficiency $\eta_{a}$ vs. $\tau / T_{S}$ calculated from (8).

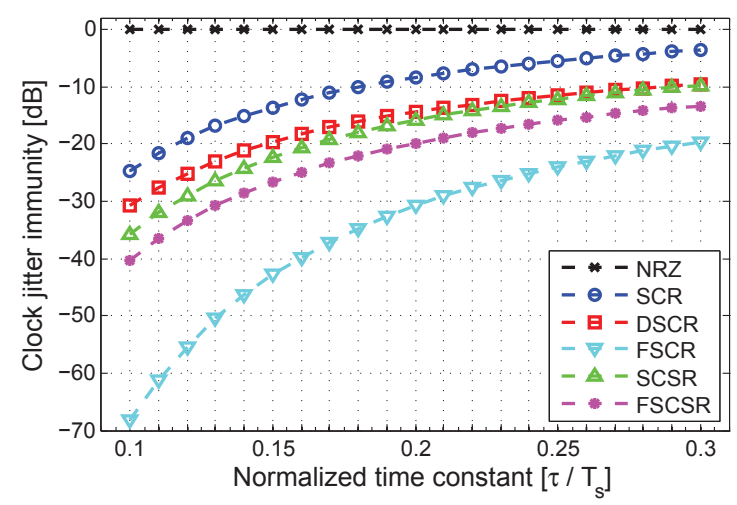

Fig. 4. The jitter immunity $\eta_{j}$ vs. $\tau / T_{S}$ calculated from (11).

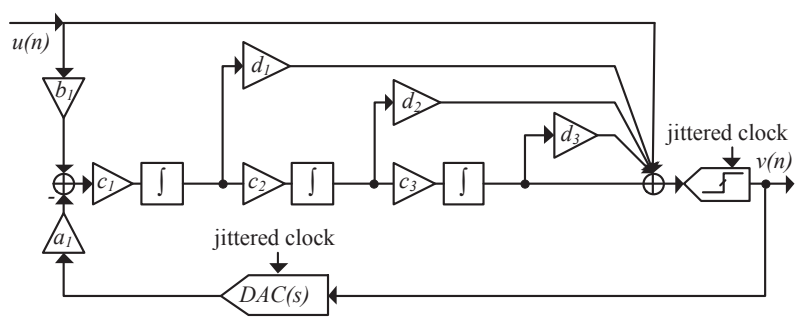

Fig. 5. Block diagram of the $3^{\text {rd }}$ order 1-bit CT $\Sigma \Delta$ modulator.

\section{Simulation Results}

To validate the theoretical results, the DACs are employed in a $3^{\text {rd }}$ order 1-bit CT $\Sigma \Delta$ modulator and behavioral simulations are performed using Matlab/Simulink. The modulator has an OSR $=80$ and its topology is illustrated in Fig. 5. The CT loop filter coefficients $c_{1}, c_{2}$ and $c_{3}$ are derived for different feedback DACs by using the impulse-invariant transformation. In order to obtain a jitter limited performance, apart from the white clock jitter, other modulator non-idealities such as excess loop delay and process variation are excluded in the model.

The CT $\Sigma \Delta$ modulator with different feedback DACs is simulated under clock jitter influence. The amount of jitter is varied in the range of $\sigma_{j}=0.1 \sim 10 \% T_{S}$. The testing 
signal is at $-6 \mathrm{dBFS}$ and its frequency is around $f_{B} / 4$. Since the time constant $\tau$ directly determines the jitter sensitivity [2][3], which is also indicated by Fig. 4, a moderate value of $\tau=0.2 T_{S}$ is selected in this test case. The simulated SNDR performance of the modulator employing different feedback DACs is shown in Fig. 6. Since for each run the SNDR can be slightly different due to randomly generated jitter, each data point in this plot is averaged from 10 runs of simulations. In addition, the averaged data points are presented together with their standard deviation using error bars. Fig. 6 confirms our observation from theoretical analysis that the modulator employing a FSCSR DAC is less sensitive to clock jitter than any other DACs apart from the FSCR. However, this superior jitter immunity is obtained under the assumption that only starting and ending time instants $(\alpha$ and $\beta$ ) of the pulse are jitter affected. The jitter effect on $\gamma$ is rarely treated in the CT $\Sigma \Delta$ literature. To the best of our knowledge, only in [3][8] this issue is studied which shows that large delay line jitter can be allowed without significantly decreasing the SNDR. Nevertheless, the readers should be awared that this insensitivity to the delay line jitter, $\gamma$, can be validated by both simulation and measurement results, only in their particular case. As the jitter in $\gamma$ is strongly implementation dependent, special attention is needed in implementing the FSCSR DACs.

In order to investigate the amplitude efficiency, the feedback waveform of each DAC at the $1^{\text {st }}$ integrator's input is plotted and compared in Fig. 7. For better illustration, the modulator is simulated with a jitter free clock. The time constant $\tau$ is again selected as $0.2 T_{s}$, since $\tau$ has also significant impact on the amplitude efficiency. The simulation results confirm the theoretical results by showing that the proposed FSCSR DAC only slightly increases the feedback peak amplitude compared to the NRZ, while the others have non-trivial increased amplitude. As the slew rate is highly circuit implementation dependent [6], the behavioral simulation used in this study cannot give accurate results in the slew rate estimation. However, it has been shown that reducing the peak amplitude of feedback DAC can consequently reduce the slew rate requirements for the integrating amplifiers [2][3]. This indicates that the proposed FSCSR DAC can potentially relax the stringent design requirements on the $1^{\text {st }}$ integrator compared with other exponentially decaying DACs.

\section{CONCLUSION}

In this paper, various exponentially decaying feedback DACs used in CT $\Sigma \Delta$ modulators have been thoroughly analyzed. All the SCR-based DACs have been generalized in terms of jitter induced charge error and its variance, DT transfer function, IBN, and two performance metrics, i.e., amplitude efficiency and jitter immunity. Moreover, a FSCSR feedback DAC has been proposed whose advantage in both metrics has been demonstrated mathematically and through behavioral simulations.

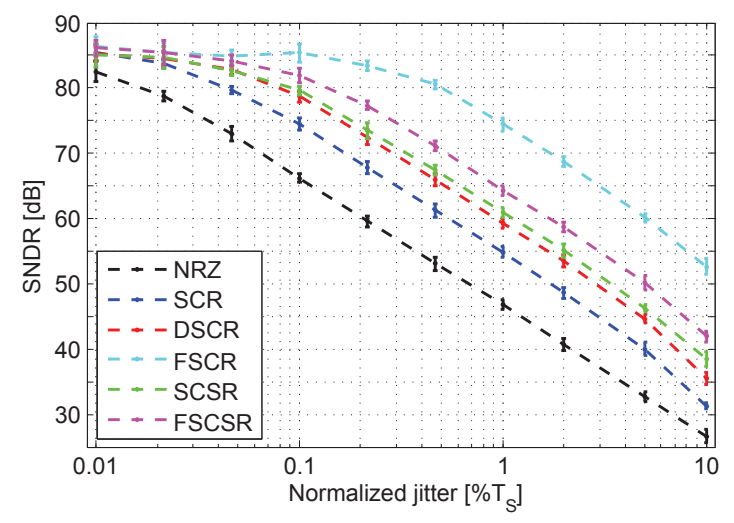

Fig. 6. Simulated SNDR of the CT $\Sigma \Delta$ modulator under clock jitter influence $\left(\tau=0.2 T_{S}\right) . P_{\text {sig }}=-6 \mathrm{dBFS}$ and $f_{\text {sig }}=f_{B} / 4$.

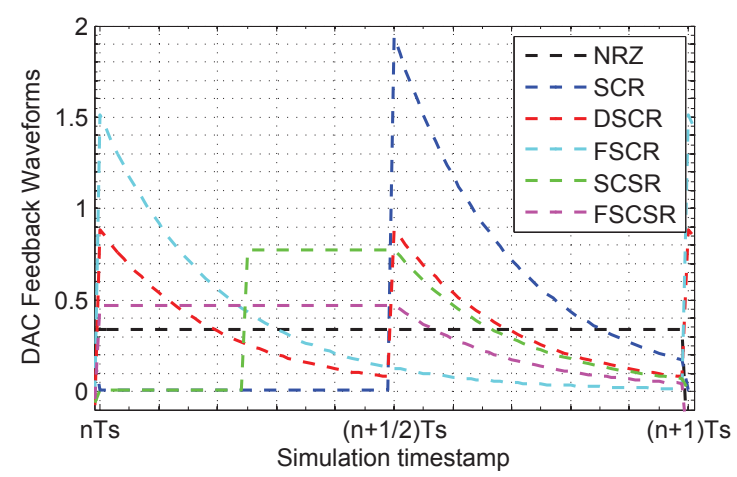

Fig. 7. DAC feedback waveforms at the $1^{\text {st }}$ integrator's input $\left(\tau=0.2 T_{S}\right)$.

\section{ACKNOWLEDGMENT}

The authors would like to thank the Swedish Research Council (VR) for funding this work.

\section{REFERENCES}

[1] J. Cherry and W. Snelgrove, Continuous-time delta-sigma modulators for high-speed $A / D$ conversion: theory, practice, and fundamental performance limits. Kluwer Academic, 2000.

[2] M. Ortmanns F. Gerfers, and Y. Manoli, "A continuous-time modulator with reduced sensitivity to clock jitter through SCR feedback," IEEE Transactions on Circuits and Systems I: Regular Papers, vol. 52, no. 5, pp. 875-884, 2005.

[3] M. Anderson and L. Sundström, "Design and Measurement of a CT $\Sigma \Delta$ ADC With Switched-Capacitor Switched-Resistor Feedback," IEEE Journal Solid-State Circuits, vol. 44, no. 2, pp. 473-483, 2009.

[4] J. G. Jo, J. Noh, and C. Yoo, "A 20-MHz Bandwidth Continuous-Time Sigma-Delta Modulator With Jitter Immunity Improved Full Clock Period SCR (FSCR) DAC and High-Speed DWA," IEEE Journal of Solid-State Circuits, vol. 46, no. 11, pp. 2469-2477, 2011.

[5] D. Radjen, P. Andreani, M. Anderson, and L. Sundström, "A continuous time modulator with reduced clock jitter sensitivity through DSCR feedback," in IEEE NORCHIP Conference, Nov. 2011, pp. 1-4.

[6] M. Ortmanns and F. Gerfers, Continuous-time sigma-delta A/D conversion: fundamentals, performance limits and robust implementations. Springer, 2006.

[7] O. Oliaei, "Clock jitter effect in continuous-time oversampling converters," in IEEE International Symposium on Circuits and Systems, May 2001, pp. 288-291.

[8] M. Anderson and L. Sundström, "A 312-MHz CT modulator using a SC feedback DAC with reduced peak current," in European Solid State Circuits Conference, Nov. 2007, pp. 240-243. 